\title{
Effect of the matrix system in the delivery and in vitro bioactivity of microencapsulated Oregano essential oil
}

\author{
Sara Beirão da Costa ${ }^{\mathrm{a}, *}$, Claudia Duarte $^{\mathrm{a}}$, Ana I. Bourbon ${ }^{\mathrm{b}}$, Ana C. Pinheiro ${ }^{\mathrm{b}}$, Ana Teresa Serra ${ }^{\mathrm{d}, \mathrm{e}}$, \\ Margarida Moldão Martins ${ }^{a}$, Maria Isabel Nunes Januário ${ }^{a}$, António A. Vicente ${ }^{b}$, Ivonne Delgadillo ${ }^{c}$, \\ Catarina Duarte $^{\mathrm{d}, \mathrm{e}}$, Maria Luísa Beirão da Costa ${ }^{\mathrm{a}}$ \\ ${ }^{a}$ CEER - Biosystems Engineering, ISA, Technical University of Lisbon, Tapada da Ajuda, 1349-017 Lisboa, Portugal \\ ${ }^{\mathrm{b}}$ IBB - Institute for Biotechnology and Bioengineering, Centre of Biological Engineering, Universidade do Minho, Campus de Gualtar, 4710-057 Braga, Portugal \\ ${ }^{\mathrm{c}}$ Chemistry Department, Research Unit QOPNA, University of Aveiro, Campo de Santiago, 3810-193 Aveiro, Portugal \\ ${ }^{\mathrm{d}}$ Instituto de Biologia Experimental e Tecnológica, Apartado 12, 2781-901 Oeiras, Portugal \\ e Instituto de Tecnologia Química e Biológica, Universidade Nova de Lisboa, Avenida da República, 2780-157 Oeiras, Portugal
}

\section{A R T I C L E I N F O}

Article history:

Available online 12 June 2011

\section{Keywords:}

Microencapsulation

Oregano essential oil

Antioxidant activity

Antimicrobial activity

Starch

Gelatine/sucrose

Inulin

\begin{abstract}
A B S T R A C T
The effect of encapsulating matrix on retention, protection and delivery of Oregano essential oil (EO) was studied. EO was encapsulated in rice starch porous spheres, inulin and gelatine/sucrose capsules by spray drying. Gelatine/sucrose matrix was also dried by freeze drying. Experimental designs were applied to test the effect of bonding agents and solids content for rice starch and drying temperature and solids content for inulin and gelatine/sucrose systems. The ratio of gelatine/sucrose was also tested. EO was identified (confocal laser scanning microscopy and FT-IR) in all tested matrices and the release profiles, antioxidant activity and antimicrobial activity of encapsulates evaluated. Results showed that the three tested materials are able to encapsulate Oregano EO. Higher diffusion coefficients were obtained for starch microcapsules (about $10^{-13} \mathrm{~m}^{2} / \mathrm{s}$ ) followed by spray-dried gelatine/sucrose systems (about $10^{-15} \mathrm{~m}^{2} / \mathrm{s}$ ) and inulin microcapsules (about $10^{-16} \mathrm{~m}^{2} / \mathrm{s}$ ). Gelatine/sucrose microparticles exhibit high antioxidant and antimicrobial activity while inulin and rice starch microencapsulates ensure higher stability.
\end{abstract}

(C) 2011 Elsevier Ltd. All rights reserved.

\section{Introduction}

There is increasing evidence that reactive oxygen species (ROS) are involved in several inflammatory and degenerative diseases, such as cancer, cardiovascular disease, type 2 diabetes and agingrelated disorders (Reuter et al., 2010; Fearon and Faux, 2009). Dietary antioxidants are believed to be powerful nutrients in the prevention of these oxidative stress related diseases (Kaur and Kapoor, 2001). These compounds present a wide range of bioactivities namely induction of endogenous antioxidant enzymes, regulation of glucose absorption, regulation of cell proliferation and differentiation, improving lipoprotein profile, modulation of inflammation, lowering blood pressure and improving homeostasis regulation (revised by Stevenson and Hurst, 2007) thus contributing to a healthy lifestyle. Therefore, through the last years, substantial efforts have been focused on the use of natural antioxidants for the

* Corresponding author. Address: CEER - Biosystems Engineering, Instituto Superior de Agronomia, Technical University of Lisbon, Tapada da Ajuda, 1349017 Lisboa, Portugal. Tel.: +351 21 3653246; fax: +351 213653200 .

E-mail address: sarabeirao@isa.utl.pt (S. Beirão da Costa). development of novel health-promoting ingredients with application in food industry.

Oregano is an aromatic plant widespread in Mediterranean countries, used as food ingredient due to its pleasant flavor. Moreover, the antioxidant (Kulisic et al., 2004; Fasseas et al., 2008) and antimicrobial (Seydim and Sarikus, 2006; Govaris et al., 2009) properties of Oregano essential oil (EO) makes this natural component also a good alternative as food preservative (Goulas and Kontominas, 2007) and health promoting substance. Those properties are mainly related to the presence of compounds such as carvacrol and thymol.

Microencapsulation is a technology that allows sensitive ingredients to be physically entrapped in a homogeneous or heterogeneous matrix aiming at their protection. The development of encapsulation delivery systems ("wall" materials) that carry, protect, and deliver functional food ingredients ("core" materials) to their specific site of action are one of the present challenges in food engineering.

The choice of the wall material is an important step for the success of the microencapsulation process. Traditionally this selection involved trial-and-error procedures in which the microcapsules 
were formed and then evaluated for encapsulation efficiency and stability (Pérez-Alonso et al., 2003).

The most popular polymers for food ingredient encapsulation include gelatine (Shu et al., 2006; Chiu et al., 2007), whey protein (Brückner et al., 2007) and carbohydrates such as starches, corn syrup solids or maltodextrins (Brückner et al., 2007; Kanakdande et al., 2007; Gharsallaoui et al., 2007).

The most widespread kinds of starch used as encapsulation agents are modified starches, from different natural sources, leading to the formation of smooth continuous wall capsules. However, small size starch granules subjected to spray-drying has the ability to form porous spherical aggregates with potential to encapsulation (Zhao and Whistler, 1994; Trindade and Grosso, 2000; Tari et al., 2003). In previous work (Beirão-da-Costa et al., 2011) rice starch spherical aggregates, produced either with or without bonding agents, were characterized in terms of particle size distribution, crystalline structure and porous structure, depending on starch and bonding agents' concentration. From the results, it was concluded that gelatine, rather than a bonding agent acts as structuring one affecting particle size, crystallinity and internal void volume of the spray-dried structures.

Flavored gelatine capsules may be used to contain a wide range of materials for use in foods (Gourdel and Tronel, 2001). It has been used for encapsulation of flavors in mixtures of carbohydrates and emulsifiers (Yoshii et al., 2001). When hydrocarbon compounds are also component of wall materials, namely together with gelatine, can act as plasticizer, promoting the formation of spherical and smooth-surfaced microcapsules, enhancing adhesion force between wall and core materials, (Bruschi et al., 2003). Reported successful examples have been the mixture of gelatine and sucrose used in microencapsulation of beta-carotene (Zhu et al., 1998). A mixture consisting of gum Arabic-sucrose-gelatine in the weight ratio of $1: 1: 1$ is an efficient encapsulant for limonene encapsulation by freeze-drying (Kaushik and Roos, 2007).

In the food field, the use of inulin as wall material is almost unexploited. Saénz et al. (2009) report the ability of inulin for microencapsulation of bioactive compounds from cactus pear fruit. Inulin is a fructooligosaccharide generally obtained from chicory (Cichorium intybus) root, Dahlia (Dahlia pinuata Cav.) and Jerusalem artichoke (Helianthus tuberosus). The inulin is composed of fructose units with $\beta(2-1)$ links with glucose at the end of the chain. These compounds are non-digestible by the human organism only being degraded by certain colon bacteria like bifidobacteria (Gibson and Kolida, 2007; Roberfroid, 2007). Additionally it has a dietary fiber action and improves calcium bioavailability (Saénz et al., 2009).

The present work aims the evaluation of bioactivity of Oregano essential oil encapsulated in three different matrices: rice starch microspherules, gelatine/sucrose microcapsules and inulin microcapsules, produced by spray and/or freeze drying. In order to access the antioxidant activity of EO microparticles, two chemical assays were performed. The antimicrobial activity of Oregano EO microcapsules was assessed against five pathogenic bacteria and one yeast.

\section{Materials and methods}

\subsection{Materials}

Oregano (Origanum vulgare L.) was produced in Alentejo region in the south of Portugal. The essential oil was obtained by steam distillation of dried flowers and leaves and stored in the dark at refrigeration prior to the encapsulation process. Gelatine (120 bloom) and sucrose (MW $342.30 \mathrm{~g} / \mathrm{mol}$ ), were purchased from Panreac Quimica S.A. A commercial inulin ( $>90 \%$ inulin; DP $\geqslant 10$ )
(Raftiline ST), obtained from chicory roots was kindly supplied by Beneo-Orafti (Tienen, Belgium).

A commercial source of rice starch $(10.6 \pm 0.78 \%$ humidity; $0.6 \pm 0.13 \%$ protein; $0.06 \pm 0.008 \%$ total fat; $28.0 \pm 0.35 \%$ amylose) was obtained from Remy Industries (Belgium). Carboxymethylcellulose (CMC) and gelatine (80-100 blooms) were obtained, respectively, from Fluka (Sweden) and PANREAC (Spain).

Chemicals used for antioxidant activity assays were: 2',2'-Azobis (2-amidinopropane) dihydrochloride (AAPH), caffeic acid, cobalt fluoride tetrahydrate (CoF2) hydrogen peroxide $\left(\mathrm{H}_{2} \mathrm{O}_{2}\right)$ and 6-hydroxy-2,5,7,8-tetramethylchroman-2-carboxylic acid (Trolox) from Sigma-Aldrich (St. Quentin Fallavier, France). Disodium fluorescein (FL) was obtained from TCI Europe (Antwerp, Belgium). Phosphate buffer solution (PBS) was prepared in distilled water with sodium chloride, potassium chloride and potassium phosphate, all from Sigma-Aldrich (St. Quentin Fallavier, France), and sodium phosphate dibasic dehydrate from Riedel-de-Haën (Seelze, Germany).

In antimicrobial assays, culture media used were Tryptone Soya broth (TSB) and Tryptone Soya agar (TSA) purchased from Oxoid IncChemicals (Basingstoke, England).

\subsection{Methods}

\subsubsection{Preparation of microparticles}

2.2.1.1. Spray drying. Spherical microparticles were produced by spray drying of matrix/EO emulsions in a LabPlant SD-04 (Leeds, UK) spray dryer equipped with a $0.5 \mathrm{~mm}$ diameter nozzle. The pressure of compressed air for the flow of spray was adjusted to 1.9 bar. The inlet temperature was set according to each experimental design. A peristaltic pump feed the spray dryer.

Rice starch was dispersed in water at room temperature (about $22^{\circ} \mathrm{C}$ ) and the mixture was homogenized thoroughly for $30 \mathrm{~min}$ under magnetic stirring. The addition of different bonding agents (CMC and gelatine) was tested. When bonding agents were added, those were previously dissolved in water at $40^{\circ} \mathrm{C}$. Gelatine and sucrose were dissolved in distilled water at $30^{\circ} \mathrm{C}$ subjected to magnetic stirring $(400 \mathrm{rpm})$ for $30 \mathrm{~min}$. Solutions of inulin were prepared in hot water $\left(80^{\circ} \mathrm{C}\right)$.

Oregano essential oil (EO), extracted by steam distillation, was added to cooled wall material solutions/suspensions at a constant $15 \%(\mathrm{w} / \mathrm{w})$. The mixtures were strongly homogenized with an ultra-turrax homogeniser (IKA LabortechNK T25 Basic) to produce stable emulsions.

2.2.1.2. Freeze drying. Gelatine/sucrose microparticles were also prepared by freeze drying. The emulsions were prepared by a similar way (Section 2.2.1.1).

\subsubsection{Experimental design}

Feed suspensions/solutions were prepared from different formulations according to an experimental design. Rice starch microparticles were prepared according a $2^{(\mathrm{k}-\mathrm{p})}$ fractional factorial design. The tested independent factors were starch concentration $(20-35 \% \mathrm{w} / \mathrm{w})$, gelatine concentration $(0-1 \% \mathrm{w} / \mathrm{w}$ starch $)$ and CMC concentration ( $0-1 \% \mathrm{w} / \mathrm{w}$ starch) in a total of nine runs. For preparation of inulin and gelatine/sucrose microparticles central composite rotatable designs were applied. Inulin microcapsules were prepared varying inulin concentration $(5-25 \% \mathrm{w} / \mathrm{w})$ and drying temperature $\left(120-190^{\circ} \mathrm{C}\right)$. Gelatine/sucrose microparticles were also prepared from different solids $(5-10 \% \mathrm{w} / \mathrm{w})$ and gelatine $(20-80 \% \mathrm{w} / \mathrm{w})$ concentrations. For the spray dried system the drying temperature was also changed $\left(120-190{ }^{\circ} \mathrm{C}\right)$.

The dependent variables analyzed were, for all tested matrices, those described in Section 2.2.3. 


\subsubsection{Analysis of microparticles}

2.2.3.1. Particle size analysis. The starch spherical aggregates size distribution was analyzed by laser light scattering (Mastersizer X, Malvern, UK) with a $300 \mathrm{~mm}$ lens. Starch powders were dispersed under continuous mechanical agitation in isopropyl alcohol until an obscuration of $11-15 \%$ was attained. Three replicates of duplicate samples $(n=6)$ measurements were recorded at 2 min intervals.

Gelatine and inulin microparticles size distribution were calculated from the measures of capsules diameters with SEMAFORE software (v 5.0). Around 1000 measurements were performed for each sample.

2.2.3.2. Confocal laser scanning microscopy. Confocal laser scanning microscopy (Olympus Fluoview, FV 1000) was used to visualize the encapsulated oil phase of the microcapsules. For visualization of the oil in the microcapsules, fluorescent $\beta$-carotene was dissolved in the oil phase prior spray-drying. Once $\beta$-carotene shows a strong fluorescence, a concentration of $0.1 \%$ (w/w EO basis) was required. The laser was adjusted in green or blue fluorescence mode which yielded two excitation wavelengths at 488 and $350 \mathrm{~nm}$, respectively. The superposition of the images obtained in these two channels allowed visualizing the oil phase and the capsule components in the same image.

2.2.3.3. Fourier transform infrared spectroscopy (FT-IR). The FT-IR spectra of samples were obtained using a Golden Gate single reflection diamond ATR system in a Brucker IFS-55 spectrometer. The spectra were recorded from 4000 to $600 \mathrm{~cm}^{-1}$ (mid infrared region) at the resolution of $8 \mathrm{~cm}^{-1}$. Five replicate spectra (256 co-added scans) were collected for each sample. The spectra were converted to JCAMP-DX format, and analyzed for PCA by a data analysis software package (Barros, 1999). After selecting the optimal wavenumbers for each sample, each spectrum, was autoscaled (centered and divided by the standard deviation). In the case of starch the spectral range used was $3465-600 \mathrm{~cm}^{-1}$ (the $\mathrm{CO}_{2}$ region between 2400 and $2290 \mathrm{~cm}^{-1}$ was deleted).

2.2.3.4. Release of EO from microcapsules. Oregano essential oil release profiles were obtained by dialysis (Nastruzzi, 1999). $100 \mathrm{mg}$ of microcapsules were added into a dialysis membrane (molecular weight cut-off 3500; Cellu-Sep H1, Membrane filtration products, USA); the membrane was subsequently placed into $100 \mathrm{~mL}$ of phosphate buffer solution with magnetic stirring. At regular time intervals, $1 \mathrm{~mL}$ samples were taken from the PBS and EO concentration was followed as a function of time by measuring the absorbance (Elisa Biotech Synergy HT) at $277 \mathrm{~nm}$, which corresponds to the maximum absorbance peak of carvacrol which is the major compound (66.4\% in weight) present in Oregano EO used in this study. Three replicates of the experiments were performed.

Kinetics of release: The diffusion of low molecular weight compounds in polymers matrix is generally governed by migration of the small molecules into pre-existing formed spaces between polymer chains (Del Nobile et al., 1994). In diffusion-controlled release of Oregano EO entrapped in spherical microparticles, the relationship between the size of the particles and the released fraction of EO at time $t$ can be described by well-known equations (Prata et al., 2008; Romero-Cano and Vincent, 2002; Zhang et al., 2006):

$\frac{M_{t}}{M_{\infty}}=6\left(\frac{D t}{\pi r^{2}}\right)^{0.5}-\frac{3 D t}{r^{2}}$ for $\frac{M_{t}}{M_{\infty}} \leq 0.7$

and

$\frac{M_{t}}{M_{\infty}}=1-0.61 \exp \left(-\frac{D t \pi^{2}}{r^{2}}\right)$ for $\frac{M_{t}}{M_{\infty}} \geq 0.7$ where $M_{t}$ is the solute mass released at time $t ; M_{\infty}$ the solute mass released at infinite time when equilibrium is achieved; $r$ is the radius and $D$ can be calculated from the Eq. (1) or Eq. (2) by non-linear parameter estimation.

In order to evaluate the changes in the system (diameter, erosion/degradation) during the release experiments, some preliminary experiments were carried: a sample of microcapsules was collected from dialysis membrane and it was observed by optical microscopy that only in the first seconds of release (immediately after microcapsules are introduced in the aqueous medium) a significant change on diameter have occurred, after that the systems remained almost constant (data not shown). Based on these results, it was assumed that EO was predominantly released by Fickian diffusion. Therefore Fick's law diffusion for spherical particles was applied assuming that: (1) the EO is dispersed uniformly throughout the matrix; (2) the EO release rate is governed by the penetration of the solvent into the monolithic matrix controlled system; (3) there was no contribution from convection; (4) the EO concentration in the release media remains close to zero (very dilute solutions) and (5) microparticles' dimensions remain constant, considering that variations in dimensions were instantaneous when the microcapsules were introduced into the dissolution medium.

2.2.3.5. Antioxidant activity. Sample preparation: Before antioxidant activity experiments, EO microencapsulated systems were prepared in DMSO followed by a gentle vortex agitation. This solvent assures the destruction of the microparticles formed and therefore the complete delivery of the essential oil to the medium. The final concentration of each system was $50 \mathrm{mg} / \mathrm{mL}$.

Oxygen radical absorbance capacity (ORAC) assay: ORAC assay was used to evaluate the antioxidant capacity of the samples towards peroxyl radicals. The assay measures the ability of the antioxidant species present in the sample to inhibit the oxidation of FL catalyzed by AAPH - generated peroxyl radicals (ROO.) and was carried using a modified method of Ou et al. (2001), previously described by Serra et al. (2011). Briefly, the composition of the reaction mixture was $1.5 \times 10^{-7} \mathrm{mM} \mathrm{FL}, 1.9 \times 10^{-1} \mathrm{M}$ AAPH (prepared in $75 \mathrm{mM}$ PBS, pH 7.4) and the appropriate diluted samples, making up a total volume of $200 \mu \mathrm{L}$. This mixture was placed in a fluorescent 96 -well microplate at $37^{\circ} \mathrm{C}$, and the reaction was started by the addition of AAPH. Fluorescence emitted by the reduced form of FL was measured in an FL800 reader (Bioteck Instruments, USA) and recorded every $1 \mathrm{~min}$ at the emission wavelength of $530 \pm 25 \mathrm{~nm}$ and excitation wavelength of $485 \pm 20 \mathrm{~nm}$ for a period of $30 \mathrm{~min}$. Phosphate buffer was used as a blank and solutions of $10,20,30,40$, and $50 \mu \mathrm{mol} / \mathrm{L}$ of Trolox were used as control standards. Final ORAC values were calculated by a regression equation between the Trolox concentration and the net area under the FL decay curve and were expressed as Trolox equivalents antioxidant capacity per $\mathrm{g}$ of microcapsules ( $\mu \mathrm{mol} \mathrm{TEAC} / \mathrm{g})$. Results presented are a mean of six replicates.

Hydroxyl radical adverting capacity (HORAC) assay: HORAC assay was based on a previously reported method (Ou et al., 2002), modified for the FL800 microplate fluorescence reader (Bio-Tek Instruments, Winooski, VT, USA) as described by Serra et al. (2011). This assay evaluates the hydroxyl radical prevention capacity of a sample using fluorescein (FL) as the probe. The hydroxyl radical was generated by a $\mathrm{Co}$ (II)-mediated Fenton like reaction and, similarly to ORAC assay, the fluorescence decay curve of FL was used to quantify the HORAC value. Briefly, $10 \mu \mathrm{L}$ of appropriate dilutions of samples were added to $180 \mu \mathrm{L}$ of FL $\left(4 \times 10^{-3} \mu \mathrm{M}\right)$ plus $5 \mu \mathrm{L}$ of $\mathrm{CoF}_{2}(1.57 \mathrm{mg} / \mathrm{mL})$. The reaction was started by the addition of $5 \mu \mathrm{L} \mathrm{H}_{2} \mathrm{O}_{2}(1.1 \mathrm{M})$ to the mixture placed in a 96-well microplate at $37^{\circ} \mathrm{C}$. Fluorescence emitted by the reduced form of FL was mea- 
sured and recorded every 1 min during 35 min. The FL800 microplate fluorescence reader was used with fluorescence filters for an excitation wavelength of $485 \pm 20 \mathrm{~nm}$ and an emission wavelength of $530 \pm 25 \mathrm{~nm}$, and the plate reader was controlled by software Gen5. Caffeic acid was used as a standard as it provides a wider linear range as compared to gallic acid. Data was expressed as micromoles of caffeic acid equivalents (CAE) per gram of extract. Results presented are a mean of six replicates.

2.2.3.6. Antimicrobial activity. Microorganisms: Microorganisms used in this study include five bacteria, including four Gram-positive strains (Listeria monocytogenes ATCC15313, Bacillus cereus ATCC14579, Staphylococcus aureus ATCC6538 and Enterococcus faecalis ATCC19433) and one Gram-negative bacteria (Escherichia coli ATCC10798), and one yeast (Saccharomyces cerevisiae ATCC10231) all of them obtained from American Type Culture Collection (ATCC) (Manassas, VA).

Microdilution assay: Microdilution assay was used to determine the antimicrobial activity of the Oregano essential oil. Briefly, the wells of a 96-well microplate were filled with $100 \mu \mathrm{L}$ of microorganism growing culture plus $100 \mu \mathrm{L}$ of various essential oil concentrations diluted in TBS (final concentrations range between 0 and $1 \mathrm{mg} / \mathrm{mL}$ ). The inoculum tested was $10^{6} \mathrm{cfu} / \mathrm{mL}$ which was determined according with calibration curves (Absorbance $600 \mathrm{~nm}$ versus microbial counts) performed previously. Then, plates were aerobically incubated during $24 \mathrm{~h}$ at $37^{\circ} \mathrm{C}$ for E. coli, S. aureus, E. faecalis, L. monocytogens or at $25^{\circ} \mathrm{C}$ for $B$. cereus and yeasts strains. The microorganism growth was followed by measuring the absorbance before and after incubation at $600 \mathrm{~nm}$ in a PowerWave Microplate Spectrophotometer (Bio-Tek Instruments, Winooski, VT, USA). The results obtained were expressed in terms of \%nhibition which is calculated according to Eq. (3)

\%Inhibition $=\left(1-\frac{\Delta A_{\text {OreganoEO+inoculum }}-\Delta A_{\text {OreganoEO }}}{\Delta A_{\text {control }}}\right) \times 100$

where $\Delta A$ is the difference of the absorbance measured at 24 and $0 \mathrm{~h}$. The minimum inhibitory concentration (MIC), which is the minimum level of essential oil concentration that produces a $90 \%$ reduction in the growth of microbial colonies, was also determined.

Plate count assay: The plate count assay was performed to evaluate the antimicrobial activity of EO capsules. Briefly, a determined quantity of essential oil ( $7.5 \mathrm{mg})$, particles $(50 \mathrm{mg}$ ) or carrier material $(50 \mathrm{mg})$ were dissolved in $10 \mathrm{ml}$ bacteria suspension $\left(10^{6} \mathrm{cfu} /\right.$ $\mathrm{mL}$ ) in TSB medium and incubated at $37^{\circ} \mathrm{C}$ for E. coli, S. aureus, E. faecalis, L. monocytogens or at $25^{\circ} \mathrm{C}$ for $B$. cereus and yeasts strains during $24 \mathrm{~h}$ with a gentle agitation $(100 \mathrm{rpm})$. The samples concentration tested was chosen taking into account the EO quantity required to inhibit approximately $40-90 \%$ of microbial growth $(0.75 \mathrm{mg} / \mathrm{mL})$.

Control was performed with bacteria suspension only. After incubation, appropriate dilutions of each culture in physiologic solution were done and spread on the surface of TSA plates. Microorganism colonies were counted after $24 \mathrm{~h}$ of incubation in order to calculate the percentage of inhibition of each system in relation to the control (growing culture without samples).

\subsubsection{Statistics}

All statistical analyses were carried out using Statistica ${ }^{\circledR} 7$ (Statsoft, Tulsa, OK, USA).

Data were fitted to second-order polynomial Eq. (1) for each dependent $\left(Y_{n}\right)$ variable, through a stepwise multiple regression analysis.

$$
\begin{aligned}
Y= & b_{0}+b_{1} X_{1}+b_{11} X_{1}^{2}+b_{2} X_{2}+b_{22} X_{2}^{2}+b_{3} X_{3}+b_{3} X_{3}^{2} \\
& +b_{12} X_{1} X_{2}+b_{13} X_{1} X_{3}+b_{23} X_{2} X_{3}
\end{aligned}
$$

where $b_{0, . . n}$ are constant regression coefficients and $X_{1, . . n}$ are independent variables.

Analysis of variance was performed to determine the lack of fit and the significance of the effects of each of the independent factors.

\section{Results and discussion}

\subsection{Encapsulation of Oregano essential oil}

Visualizing the deposition/distribution of the involved polymers and confirming the encapsulation of the bioactive compounds in controlled release systems are important steps on microencapsulation methodology. Confocal laser scanning microscope (CLSM) has been used to detect the presence of several compounds in different systems as e.g. in microcapsules. In CLSM, the light from out-of-focus structures is faded out, and at the same time this technique enables a non-destructive view through the capsule wall. By using different fluorescence labels, the unambiguous identification of several compounds is possible.

In the present work, images obtained by CLSM allow identifying the localization of EO in different microcapsules' matrices. Two fluorescence channels (blue and green lasers) were used to excite the capsule constituent polymer and the oil phase (EO + $\beta$-carotene), respectively. When excited under the blue laser it was observed that both polymer and the oil phase exhibited fluorescence, while under the green laser only the oil phase showed fluorescence.

Fig. 1(I, II and III) are superposition's of the images from the blue and green lasers, which allows distinguishing between encapsulated oil and the base structure of the microcapsules. These images show that most of the bright green spots (oil phase) are entrapped in blue regions (capsule constituent polymer). This clearly shows that the EO is homogenously distributed within the shell material.

FT-IR analyses confirm the presence of essential oil in all entrapment systems. Principal component analyses of FT-IR spectra allowed a better understanding and clearly show different groups according to matrices formulae and presence or absence of EO. As example, for rice starch system the three first principal components (PC's) explained $94.7 \%$ of total variance of the data. The scores plot from PC1 vs PC2 and PC1 vs PC3 showed in both cases that the samples are organized in two main groups with starch spherules in negative PC1 and starch spherules with encapsulated EO in positive PC1 (plots not shown).

\subsection{Release measurements}

Controlled release may be defined as a method by which an active agent or ingredient is made available at a desired site and time and at a specific rate (Pothakamury and Barbosa-Cánovas, 1995). In order to evaluate the effect of polymeric matrix in EO release, different encapsulating materials (gelatine/sucrose, starch and inulin) were tested.

The effect of polymeric matrix composition on EO release rate was evaluated by Eq. (1) and Eq. (2) in terms of diffusion coefficients. Fig. 2 shows an example of the fitting of Eq. (1) to the experimental data of EO release kinetics, for different polymeric microcapsules (starch, spray-dried gelatine/sucrose and inulin systems). A fairly good fit of the model to the experimental data can be clearly observed $\left(0.90<R^{2}<0.97, \quad 0.66<R^{2}<0.97\right.$ and $0.77<R^{2}<0.98$ for starch, spray-dried gelatine/sucrose and inulin systems, respectively, and residues normally distributed).

The effect of starch microparticles composition (starch and gelatine content) in diffusion coefficients, obtained by fitting Eq. (1) and Eq. (2) to experimental data, can be observed in Fig. 3a). The 

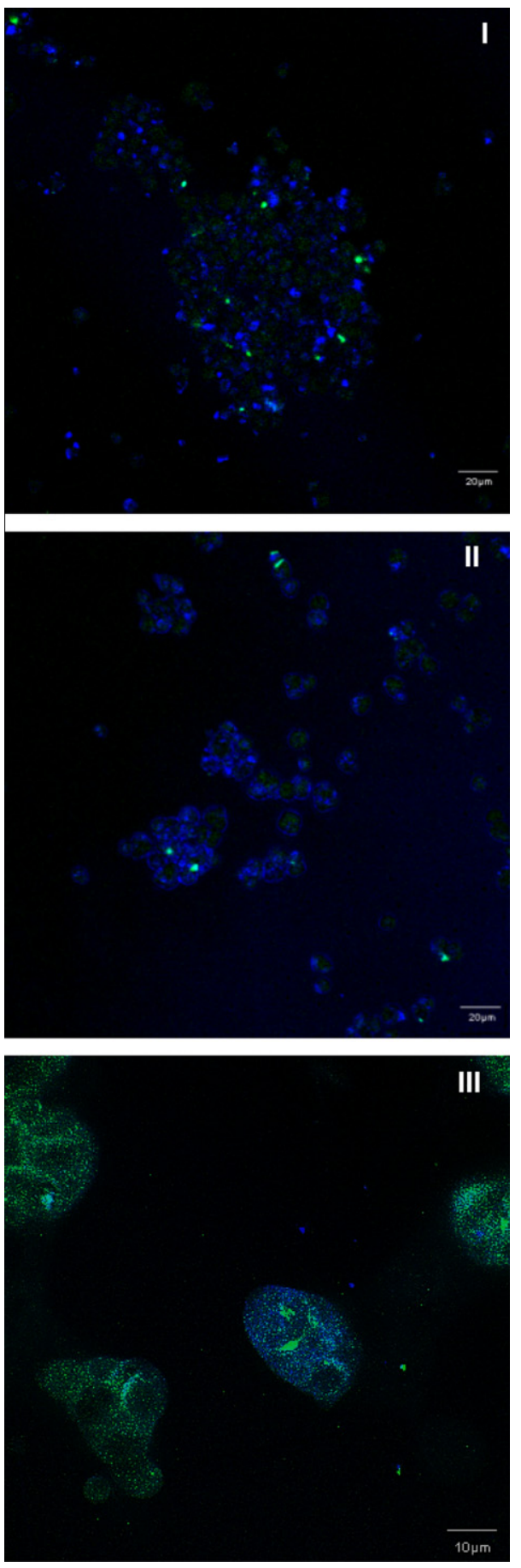

Fig. 1. Confocal laser scanning microphotographs of the microcapsules containing $\beta$-carotene-stained oil phase (EO + $\beta$-carotene) by using the blue laser and the green laser for microcapsules systems with: (I) starch $20 \%+$ EO 15\%; (II) inulin 15\% + EO $15 \%$ and (III) spray-dried gelatine $100 \%+$ TSS $7.5 \%$ + EO $15 \%$, dried at $155{ }^{\circ} \mathrm{C}$.

diffusion coefficient (ranging between $(1.99 \pm 0.01) \times 10^{-13}$ and $\left.(6.81 \pm 0.01) \times 10^{-13} \mathrm{~m}^{2} / \mathrm{s}\right)$ was mainly influenced by gelatine concentration $(p=0.01)$. Higher concentrations of this bonding agent lead to a faster release of EO. Additionally, EO release profiles from these structures evidence that when gelatine is present the total amount of released EO is higher. In previous works (Beirão-daCosta et al., 2011) it was verified that the presence of gelatine leads to an increase of void volume in the formed spheres and so these structures could have a higher potential for encapsulation. This
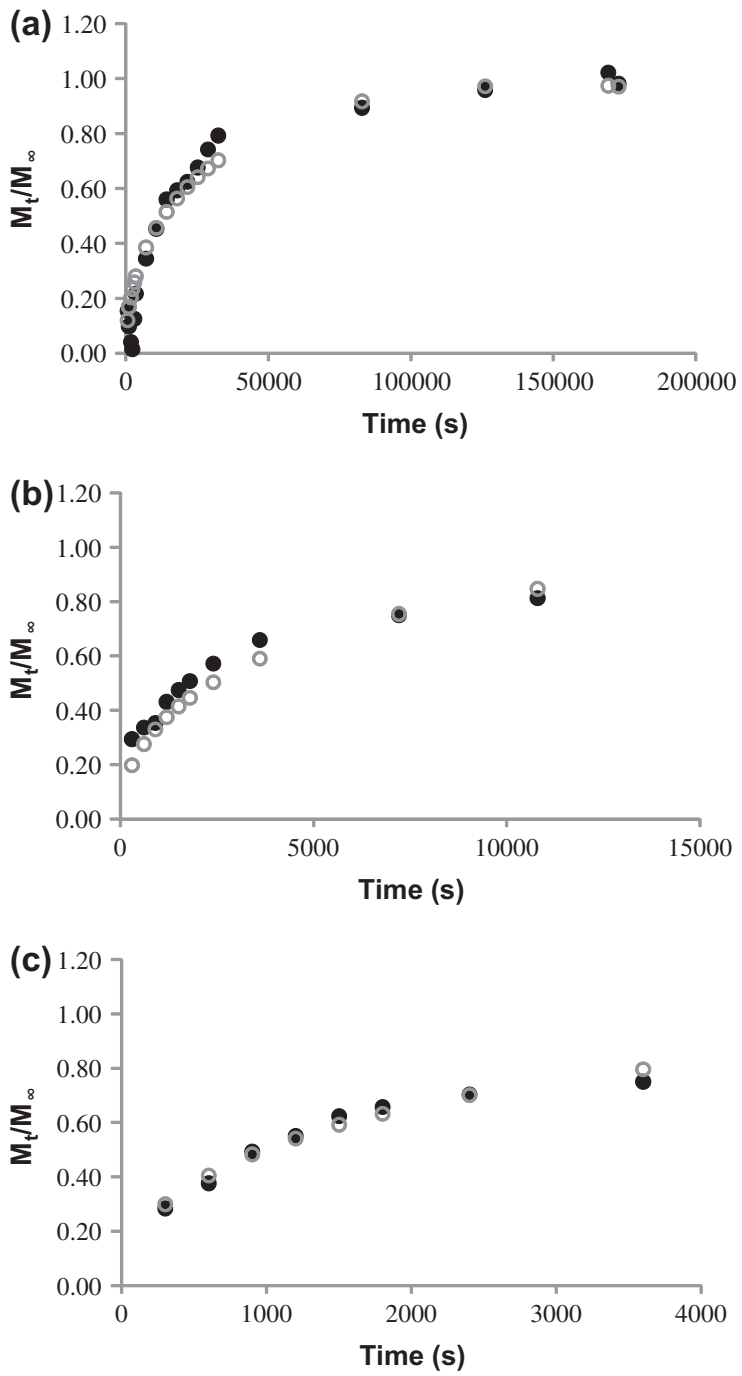

Fig. 2. Fitting of Eq. (1) to EO controlled release experimental data from microcapsules with: (a) $20 \%$ starch $+1 \%$ CMC; (b) $100 \%$ gelatine and prepared with a drying temperature of $190{ }^{\circ} \mathrm{C}$ and (c) $5 \%$ inulin and prepared with a drying temperature of $155^{\circ} \mathrm{C}$ (experimental results (๑); model-generated values $(\bigcirc)$ ).

hypothesis, in the case of Oregano EO, is confirmed by release results.

The diffusion coefficients obtained for inulin microcapsules ranged between $(2.80 \pm 0.29) \times 10^{-16}$ and $(8.76 \pm 0.61) \times 10^{-16} \mathrm{~m}^{2} / \mathrm{s}$. Fig. $3 b$ shows the effect of polymer concentration $(p>0.05)$ and drying temperature $(p<0.05)$ on the diffusion properties of the EO. Generally, a decrease of diffusion coefficient was observed for samples dried above $140{ }^{\circ} \mathrm{C}$, and can be explained by a possible thermal degradation at high temperatures that causes a volatilization or degradation of heat-sensitive ingredients. This behavior was previously reported by other authors (Gharsallaoui et al., 2007; Gouin, 2004). Also, for microcapsules dried at temperatures above $140{ }^{\circ} \mathrm{C}$, the soluble solids content did not affect the diffusion coefficient of Oregano EO. On the other hand, for microcapsules prepared at high temperatures $\left(>155^{\circ} \mathrm{C}\right)$, the effect of soluble solids on the diffusion coefficient of Oregano EO was more evident, suggesting that particle structure changes occurred, thus resulting in different diffusion coefficients.

The release experiments using gelatine/sucrose systems allowed concluding that both spray-dried and freeze-dried systems were effective in $\mathrm{EO}$ retention and release.

The diffusion coefficients found for the spray-dried gelatine/sucrose microcapsules ranged between $(0.317 \pm 0.04) \times 10^{-16}$ and 

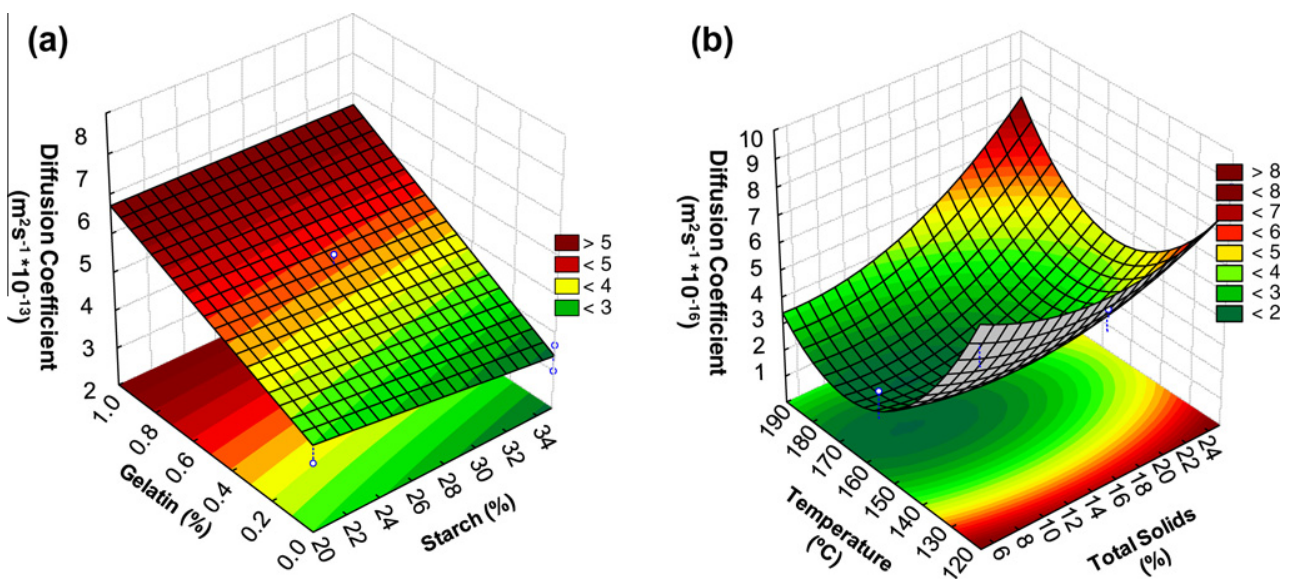

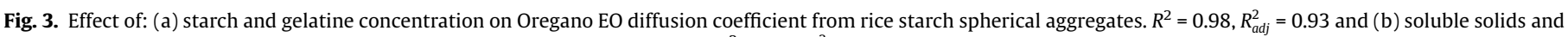
drying temperature on Oregano EO diffusion coefficient from inulin capsules. $R^{2}=0.92, R_{a d j}^{2}=0.77$.

$(1.05 \pm 0.15) \times 10^{-15} \mathrm{~m}^{2} / \mathrm{s}$ depending on gelatine/sucrose ratio, total soluble solids and drying temperature applied on microcapsules preparation. However, no correlation could be found between the diffusion coefficients and the different conditions applied during microcapsules production. Fig. 4 shows EO release kinetics from gelatine/sucrose systems. The release curves were expressed by EO mass release in relation of mass of microparticles used versus time. As can be seen, the gelatine/sucrose microcapsules displayed different EO release profiles, independent of matrix compounds concentration and the drying temperature applied on preparation of spray dried systems (Fig. 4a). The response surface model could
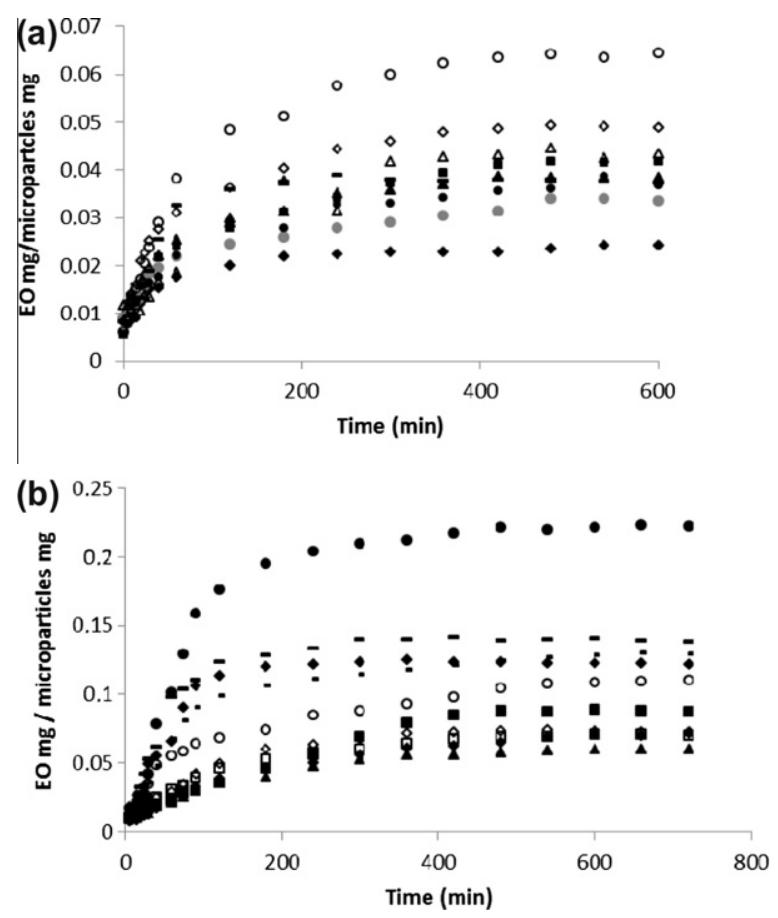

Fig. 4. Oregano EO release profiles at $25^{\circ} \mathrm{C}$ from spray-dried gelatin/sucrose microcapsules (a): ( ) $20 \%$ gelatin $/ 5 \% \mathrm{TSS} / 120{ }^{\circ} \mathrm{C}$; ( $) 100 \%$ gelatine $/ 5 \% \mathrm{TSS} / 120{ }^{\circ} \mathrm{C}$; $(\Delta) 100 \%$ gelatine $/ 5 \% \mathrm{TSS} / 190{ }^{\circ} \mathrm{C}$; (O) $100 \%$ gelatine $/ 10 \% \mathrm{TSS} / 120{ }^{\circ} \mathrm{C} ;(\diamond) 100 \%$ gelatine $/ 10 \% \mathrm{TSS} / 190{ }^{\circ} \mathrm{C}$; $(\bullet) 20 \%$ gelatine $/ 10 \% \mathrm{TSS} / 120{ }^{\circ} \mathrm{C} ;(\Delta) 60 \%$ gelatine $/ 7.5 \%$ $\mathrm{TSS} / 190{ }^{\circ} \mathrm{C}$; $(\bullet) 60 \%$ gelatine/7.5\% TSS $/ 155^{\circ} \mathrm{C} ;(-) 60 \%$ gelatine $/ 7.5 \% \mathrm{TSS} / 155^{\circ} \mathrm{C}$ and from freeze-dried microcapsules (b): ( $)$ ) 20\% gelatine/5\% TSS; (घ) $100 \%$ gelatine $/ 5 \%$ TSS; ( $\Delta$ ) 20\% gelatine/10\% TSS; $(\diamond)$ 100\% gelatine/10\% TSS; $(\bigcirc)$ 60\% gelatine/5\% TSS; (•) $60 \%$ gelatine/10\% TSS; ( $\square$ ) $20 \%$ gelatine/7.5\% TSS; (-) $100 \%$ gelatine/7.5\% TSS; (-) $60 \%$ gelatine $/ 7.5 \%$ TSS; $(\bullet) 60 \%$ gelatine/7.5\% TSS. not be applied as it was not possible to fit Eq. (1) and Eq. (2) to experimental data of some samples. Some microcapsules did not show EO release profiles possibly due to their destruction (solubilization) on the release media and, consequently leading to an instantaneous release of Oregano EO. When sucrose is present in the system, the samples were more unstable, with high hygroscopicity, both in the diffusion medium and during storage. These samples therefore are inappropriate for protection and controlled release of the core material.

Once more, it can be observed that Oregano EO release profiles showed a biphasic modulation characterized by an initially relatively rapid release period (a "burst effect") followed by a slower release phase (a "lag time"). It also can be observed that the released amount of EO from freeze-dried structures (Fig. 4b) was about 3-fold higher than for spray-dried microcapsules. This result can be explained based on the fact that the retention of Oregano EO was more effective for freeze dried microcapsules once that the process temperatures applied in the spray-drying method lead unavoidably to some loss of volatile compounds and consequently less EO was released.

Additionally, microparticles prepared by freeze-drying were more stable and this is consistent with the fact that this process is one of the most useful for drying thermosensitive substances that are unstable in aqueous solutions, such as gelatine/sucrose systems (Madene et al., 2006). Buffo and Reineccius (2001) compared spray drying, tray drying, drum drying and freeze drying to encapsulate cold-pressed Valencia orange oil with gum acacia and modified food starch. They concluded that freeze drying is the process that gives the most desirable properties to dried powder. Minemoto et al. (1997) compared oxidation of menthyl linoleate when encapsulated with either gum arabic by hot air drying and freeze drying. These authors showed that freeze drying was better than hot air drying. Indeed, this drying technique is less attractive than others because the costs of freeze drying are up to 50 times higher than spray drying (Desobry et al., 1997) and the storage and transport of particles produced is extremely expensive (Jacquot and Pernetti, 2003), the commercial applicability is also severely restricted by the long processing time (BarbosaCanovas and Vega-Mercado, 1996).On the other hand, the decrease in encapsulation efficiency by spray drying could be balanced by a higher protection degree due to the wall characteristics of the different structures created.

The results obtained for all these systems show that different release behaviors were obtained depending on the constituents of the microcapsules and the methodology applied to produce them. Higher diffusion coefficients were estimated for starch 
microcapsules (about $10^{-13} \mathrm{~m}^{2} / \mathrm{s}$ ) followed by spray-dried gelatine/sucrose systems (about $10^{-15} \mathrm{~m}^{2} / \mathrm{s}$ ) and inulin microcapsules (about $10^{-16} \mathrm{~m}^{2} / \mathrm{s}$ ).

\subsection{Antioxidant activity and stability of encapsulates}

In order to access the antioxidant activity of EO microparticles, two chemical assays were performed: ORAC and HORAC. These assays measure two different but equally important aspects of antioxidant properties - radical chain breaking and radical prevention. The HORAC primarily reflects metal chelating radical prevention ability, and the ORAC reflects peroxyl radical absorption capacity (Ou et al., 2002).

Oregano essential oil used in encapsulation processes has high antioxidant activity, presenting ORAC and HORAC values of 2 $080 \pm 21 \mu \mathrm{mol} \mathrm{TEAC/g}$ and $3542 \pm 65 \mu \mathrm{mol} \mathrm{CAEAC/g}$, respectively. Although no data have been reported for HORAC assay, the result obtained in ORAC is within the range of values reported in the literature (Bentayeb et al., 2009; www.health-herbal.com). In opposition, the three systems used as wall material alone have not any noticeable antioxidant activity (data not shown).

From the results it can be noticed that the encapsulated EO still maintained antioxidant activity for all tested encapsulation wall materials. For rice starch and inulin particles, HORAC values are not presented since the results obtained were not in the range of the method detection limit $(<40 \mu \mathrm{mol} \mathrm{CAE} / \mathrm{g})$.

The antioxidant activity of EO enriched rice starch microparticles is well adjusted by the response surface model $\left(R^{2}=0.98\right.$; $\left.R_{a d j}^{2}=0.93\right)$ and is influenced both by rice starch and gelatine concentration ( $p=0.04$ and $p=0.02$, respectively) (Fig. 5a). It was observed a significant decrease in antioxidant properties of these encapsulates when compared to free EO after the encapsulation procedures. However, after 6 months storage the residual activity of microparticles remains the same while unprotected essential oil showed a $75 \%$ decrease of the initial activity just after 2 month of storage.

ORAC values of inulin microcapsules varied between 13 and $67 \mu \mathrm{mol} \mathrm{TEAC} / \mathrm{g}$. The experimental results were also adjusted by RSM model $\left(R^{2}=0.87 ; R_{a d j}^{2}=0.71\right)$, inulin concentration being the single parameter that influence encapsulates antioxidant activity $(p=0.01)$. Higher inulin content leads to particles with higher ORAC values. Drying temperature did not affect antioxidant capacity of the particles (Fig. 5b).

(a)

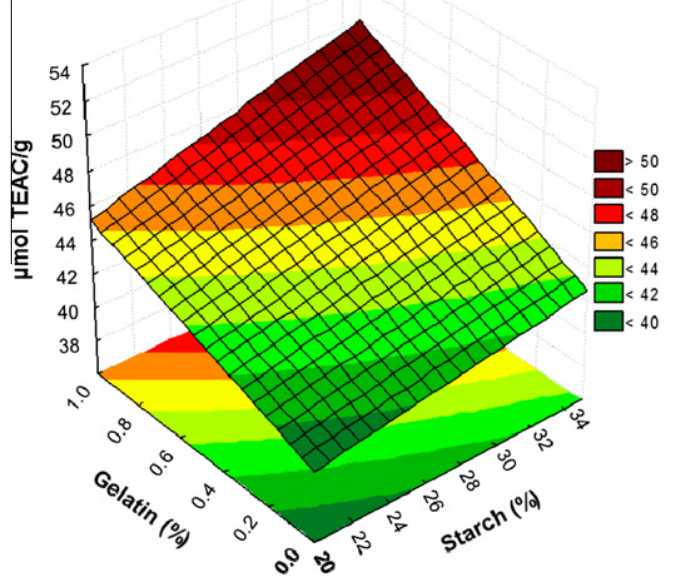

The ORAC values of all encapsulates show that gelatine/sucrose microparticles, either produced by spray or freeze drying, are those that presented highest antioxidant activity (Table 1). From the results it can be observed that gelatine content have an important role in EO protection. Some samples showed antioxidant activity values quite similar to those of free EO $(>340 \mu \mathrm{mol}$ TEAC/g). For the freeze dried system ORAC values increase with the increase in gelatine content in the mixture. This conclusion can also be extended for EO stability during storage. After 6 months storage, samples without sucrose and with $40 \%$ of sugar showed only a $12 \%$ and $13 \%$, respectively, ORAC values decrease, while samples with $20 \%$ of gelatine loss about $45 \%$ of antioxidant activity. When a pure gelatine system is used, encapsulation by spray drying did not induce a loss in particles antioxidant activity, comparing with freeze dried ones. Surprisingly, although the total amount of EO diffused from the particles is quite lower (Fig. 4) the offered antioxidant protection is similar. This feature sustains the idea that, despite a higher volatile retention achieved by freeze drying process, the protection conferred by the wall is lower conducing to a higher loss/degradation of active compounds. Drying temperature did not deleteriously affect the antioxidant properties of gelatine samples but, when sucrose is present even at $120^{\circ} \mathrm{C}$, a significant decrease in ORAC value is observed. Additionally the solids content of the initial emulsion is important in preserving the antioxidant activity of the core material.

The analysis of HORAC results confirms those of ORAC method.

\subsection{Antimicrobial activity}

Essential oils are widely recognized to have high antimicrobial activity. In particular, the essential oil derived from Oregano is already reported to inhibit the growth of several pathogenic microorganisms, such as Aspergillus niger, Aspergillus flavus, S. aureus, Salmonella typhimurium, Pseudomonas aeruginosa, B. cereus, E. coli and L. monocytogenes (Paster et al., 1990; Skandamis et al., 1999; Lambert et al., 2001; Gutierrez et al., 2008).

In this work, the antimicrobial activity of Oregano EO microparticles was assessed against five bacteria recognized as food pathogens and one yeast. In a first approach, a microdilution assay was performed in order to determine MIC values of Oregano EO. For all strains tested, the Oregano EO yielded a MIC of about $1 \mathrm{mg} /$ $\mathrm{mL}$ except for $E$. faecalis where this value was not reached at the highest concentration tested (being $>1 \mathrm{mg} / \mathrm{mL}$ ). Similarly to Busatta et al. (2007) no difference regarding sensitiveness to the EO was (b)

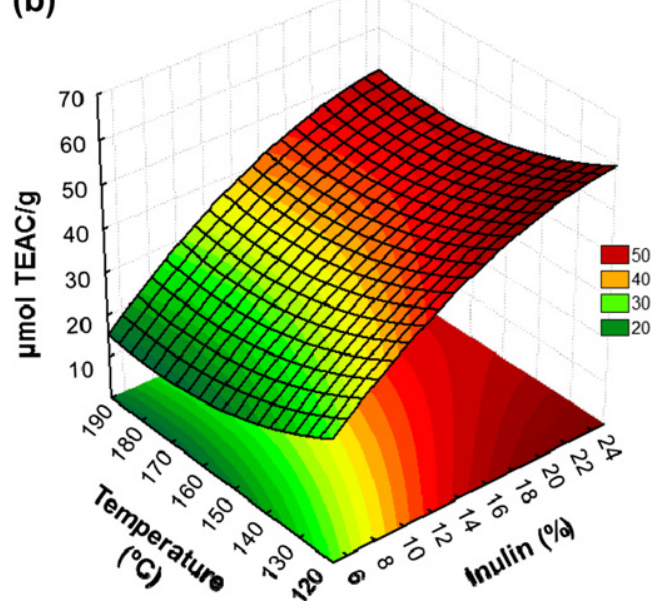

Fig. 5. Antioxidant activity of rice starch and inulin microparticles with encapsulated oregano essential oil. (a) Rice $\operatorname{starch}\left(R^{2}=0.98, R_{a d j}^{2}=0.93\right) ;(b)$ inulin $\left(R^{2}=0.87\right.$, $R_{a d j}^{2}=0.71$. 
Table 1

Antioxidant activity of gelatine/sucrose microparticles with encapsulated Oregano essential oil.

\begin{tabular}{|c|c|c|c|c|c|c|}
\hline & \multirow[t]{3}{*}{ Gelatine (\%) } & \multirow[t]{3}{*}{ Drying temperature $\left({ }^{\circ} \mathrm{C}\right)$} & \multicolumn{4}{|c|}{ Antioxidant activity } \\
\hline & & & \multicolumn{2}{|c|}{ ORAC $(\mu \mathrm{mol}$ TEAC/g $)$} & \multicolumn{2}{|c|}{ HORAC $(\mu \mathrm{mol} \mathrm{CAE} / \mathrm{g})$} \\
\hline & & & Initial & 6 Months & Initial & 6 Months \\
\hline \multicolumn{7}{|l|}{ Spray drying } \\
\hline \multirow[t]{3}{*}{$5 \%$ Total solids } & 20 & 120 & $110.1 \pm 8.7$ & $39.6 \pm 5.1$ & $152.6 \pm 27.2$ & $99.7 \pm 8.8$ \\
\hline & 100 & 120 & $211.7 \pm 15.0$ & $160.4 \pm 14.6$ & $303.1 \pm 17.0$ & $265.1 \pm 17.7$ \\
\hline & 100 & 190 & $209.4 \pm 7.9$ & $177.2 \pm 15.0$ & $320.5 \pm 15.7$ & $298.2 \pm 26.7$ \\
\hline \multirow[t]{3}{*}{ 10\% Total solids } & 60 & 155 & $247.4 \pm 21.0$ & $187.8 \pm 12.9$ & $497.3 \pm 81.1$ & $329.5 \pm 35.7$ \\
\hline & 100 & 120 & $345.7 \pm 11.5$ & $264.7 \pm 20.2$ & $409.5 \pm 17.6$ & $347.2 \pm 34.5$ \\
\hline & 100 & 190 & $325.0 \pm 0.2$ & $258.2 \pm 12.7$ & $437.3 \pm 31.5$ & $401.7 \pm 27.9$ \\
\hline \multicolumn{7}{|l|}{ Freeze drying } \\
\hline \multirow[t]{3}{*}{ 7.5\% Total solids } & 20 & - & $249.4 \pm 13.6$ & $136.9 \pm 13.0$ & $485.0 \pm 33.3$ & $291.3 \pm 22.3$ \\
\hline & 60 & - & $340.6 \pm 25.3$ & $295.5 \pm 16.7$ & $467.0 \pm 39.7$ & $437.6 \pm 40.9$ \\
\hline & 100 & - & $390.6 \pm 17.7$ & $344.2 \pm 15.1$ & $569.1 \pm 27.0$ & $483.0 \pm 21.25$ \\
\hline
\end{tabular}

observed between Gram-positive and Gram-negative bacteria. The mechanism of the antimicrobial action of Oregano EO was already reported to be related with cytoplasmic membrane disruption which further affects $\mathrm{pH}$ homeostasis and equilibrium of inorganic ions (Oussalah et al., 2007; Lambert et al., 2001). Carvacrol and thymol are pointed to be the main responsible of the Oregano EO antimicrobial effect (Lambert et al., 2001). Moreover, it is important to note that results obtained in this work are of the same order of magnitude of those found by other authors; as an example, for E. coli the values found ranged between $0.7 \mathrm{mg} / \mathrm{mL}$ (Di Pasqua et al., 2005) and $4.3 \mathrm{mg} / \mathrm{mL}$ (Ponce et al., 2003) and for L. monocytogens, $S$. aureus and $E$. faecalis the respective MIC values reported were: $0.8,1.8$ and $>10 \mathrm{mg} / \mathrm{mL}$ (Di Pasqua et al., 2005).

In order to evaluate the antimicrobial activity of EO microparticles a plate count assay was performed. Results obtained for each particle system were compared with free Oregano EO and some of them are shown in Table 2. Overall, as shown in antioxidant activity analysis, gelatine/sucrose particles were the system that conferred higher inhibitory effect against the tested microorganisms. Furthermore, the results demonstrated that all tested encapsulates did not present antimicrobial effect against B. cereus.
Concerning rice starch and inulin microencapsulates, both systems did not show inhibitory capacity against $E$. faecalis and S. cerevisiae. In particular, rice starch system was effective in inhibiting $S$. aureus and $E$. coli growth and a synergistic effect was observed when both bonding agents were present.

Inulin microcapsules provided protection against E. coli, S. aureus and L. monocytogenes. It is important to note that, drying temperature did not affect the antimicrobial activity against $L$. monocytogenes (Table 2). Nevertheless when inulin microcapsules were dried at the highest temperature $\left(190^{\circ} \mathrm{C}\right)$ no inhibition capacity was observed against $S$. aureus. Concerning $E$. coli, the antimicrobial effect of microcapsules followed a pattern similar to that observed for diffusion coefficient suggesting that the rate of release of the antimicrobial compound(s) is affecting the bacteria population growth.

For gelatine/sucrose systems, the content of gelatine in microparticles has also an important role in EO protection since antimicrobial capacity of microencapsules increased with the increase of gelatine content. In freeze dried systems, for all tested microorganisms, this effect is more evident when gelatine concentration rises from $20 \%$ to $60 \%$ than from $60 \%$ to $100 \%$. For microcapsules

Table 2

Antimicrobial activity (microorganisms growth inhibition) of microparticles with encapsulated Oregano essential oil.

\begin{tabular}{|c|c|c|c|c|c|c|c|}
\hline \multicolumn{8}{|l|}{ Inhibition (\%) } \\
\hline & & E. coli & L. monocytogenes & S. aureus & B. cereus & E. faecalis & S. cerevisiae \\
\hline \multirow{2}{*}{\multicolumn{8}{|c|}{ Starch (35\%) }} \\
\hline & & & & & & & \\
\hline CMC (\%) & Gelatine (\%) & & & & & & \\
\hline 0 & 0 & 50 & 6 & 40 & - & 16 & - \\
\hline 0 & 1 & 38 & 52 & 55 & - & - & - \\
\hline 1 & 0 & 51 & - & 65 & - & - & 15 \\
\hline 1 & 1 & 67 & 63 & 83 & 9 & 6 & - \\
\hline \multicolumn{8}{|l|}{ Gelatine/sucrose } \\
\hline \multicolumn{8}{|c|}{ Spray drying (total solids $10 \%$ ) } \\
\hline $\mathrm{G} / \mathrm{S}(\%)$ & Drying temperature & & & & & & \\
\hline $60 / 40$ & $155^{\circ} \mathrm{C}$ & 83 & 62 & - & - & 46 & 14 \\
\hline $100 / 0$ & $120^{\circ} \mathrm{C}$ & 77 & 80 & 73 & - & 97 & 99 \\
\hline $100 / 0$ & $190{ }^{\circ} \mathrm{C}$ & 96 & 51 & 51 & - & 75 & 99 \\
\hline \multicolumn{8}{|c|}{ Freeze drying (total solids $7.5 \%$ ) } \\
\hline & Gelatine (\%) & & & & & & \\
\hline & 20 & 48 & 60 & 79 & - & - & 22 \\
\hline & 60 & 95 & 99 & 99 & - & 99 & 99 \\
\hline & 100 & 100 & 87 & 100 & & 99 & 94 \\
\hline \multicolumn{8}{|l|}{ Inulin (25\%) } \\
\hline & Drying temperature & & & & & & \\
\hline & $120^{\circ} \mathrm{C}$ & 59 & 64 & 55 & - & - & - \\
\hline & $155^{\circ} \mathrm{C}$ & 29 & 61 & 64 & - & - & - \\
\hline & $190{ }^{\circ} \mathrm{C}$ & 42 & 63 & - & - & - & - \\
\hline
\end{tabular}


prepared by spray-drying, processing temperature did not affect the antimicrobial activity against $E$. coli and S. cerevisiae. Nevertheless, the inhibition of $L$. monocytogenes, S. aureus and E. faecalis was reduced at higher temperatures (Table 2 ).

These figures suggest that, the inhibition of the microorganisms' growth is conferred by the presence and concentration of specific bioactive compounds (Lambert et al., 2001). The different responses observed could be due to different thermosensitive characteristics and/or diffusion ability of those substances indicating that the carrier material, process type, and drying temperature are important factors influencing the antimicrobial activity of encapsulated Oregano EO.

\section{Conclusions}

The results obtained herein provide useful information concerning the release, bioactivity and stability of Oregano EO encapsulated in three matrices using different processing technologies. Our findings demonstrated that gelatine/sucrose microparticles exhibit high antioxidant and antimicrobial activity while inulin and rice starch microencapsulates ensure higher stability. Importantly, the bioactivity of each system could be improved by manipulating different processing parameters such as bonding agents, solids content and drying temperature.

Higher diffusion coefficients were obtained for starch microcapsules (about $10^{-13} \mathrm{~m}^{2} / \mathrm{s}$ ) followed by spray-dried gelatine/sucrose systems (about $10^{-15} \mathrm{~m}^{2} / \mathrm{s}$ ) and inulin microcapsules (about $10^{-16} \mathrm{~m}^{2} / \mathrm{s}$ ). Therefore, depending on the characteristics desired in the final product, wall materials can be selected from a wide variety of natural polymers to provide different release properties.

The knowledge gained in this study provides important insights for the design of promising functional ingredients with application in food industry.

\section{Acknowledgements}

Thanks are due for the financial support given by Portuguese Foundation for Science and Technology (FCT) through the project PTDC/AGR/ALI/67194/2006 and through a post-doctoral grant of first author (SFRH/BPD/44200/2008) supported by programme QREN - POPH - Tipologia 4.1.

\section{References}

Barbosa-Canovas, G.V., Vega-Mercado, H., 1996. Dehydratation of Foods. Chaman \& Hall, New York, NY.

Barros, A.S., 1999. Contribution à la seléction et la comparaison de variables característiques, PhD Thesis, Institut National Agronomique Paris-Grignon, (Chapter 1).

Beirão-da-Costa, S, Duarte, C., Moldão-Martins, M., Beirão-da-Costa, M.L, 2011. Physical characterization of rice starch spherical aggregates produced by spraydrying. Journal of Food Engineering 104, 36-42.

Bentayeb, K., Vera, P., Rubio, C., Nerin, C., 2009. Adaptation of the ORAC assay to the common laboratory equipment and subsequent application to antioxidant plastic films. Analytical and Bioanalytical Chemistry 394 (3), 903-910.

Brückner, M., Bade, M., Kunz, B., 2007. Investigations into the stabilization of a volatile aroma compound using a combined emulsification and spray drying process. European Food Research and Technology 226, 137-146.

Buschi, M.L., Cardoso, M.L.C., Lucchesi, M.B., Gremião, M.P.D., 2003. Gelatin microparticles containing propolis obtained by spray-drying technique: preparation and characterization. International Journal of Pharmaceutics 264, 45-55.

Buffo, R.A., Reineccius, G.A., 2001. Comparison among assorted drying processes for the encapsulation of flavors. Perfumer and Flavorist 26, 58-67.

Busatta, C., Mossi, A.J., Rodrigues, M.R.A., Casian, R.L., Oliveira, J.V.O., 2007. Evaluation of Oreganum vulgare essential oil as antimicrobial agent in sausage. Brazilian Journal of Microbiology 38, 610-616.

Chiu, Y.T., Chiu, C.P., Chien, J.T., Ho, G.H., Yang, J., Chen, B.H., 2007. Encapsulation of lycopene extract from tomato pulp waste with gelatin and poly (ç-glutamic acid) as carrier. Journal of Agricultural and Food Chemistry 55, 5123-5130.
Del Nobile, M., Mensitieri, G., Netti, P.A., Nicolais, L., 1994. Anomalous diffusion in poly-ether-ether-ketone (PEEK). Chemical Engineering Science 49 (5), 633644.

Desobry, S., Netto, F.M., Labuza, T.P., 1997. Comparison of spray-drying, drumdrying and freeze-drying for b-carotene encapsulation and preservation. Journal of Food Science 62, 1158-1162.

Di Pasqua, R., De Feo, V., Villani, F., Mauriello, G., 2005. In vitro antimicrobial activity of essential oils from Mediterranean Apiaqceae, Verbenaceae and Lamiaceae against foodborne pathogens and spoilage bacteria. Annals of Microbiology 55 (2), 139-143

Fasseas, M.K., Mountzouris, K.C., Tarantilis, P.A., Polissiou, M., Zervas, G., 2008 Antioxidant activity in meat treated with oregano and sage essential oils. Food Chemistry 106 (3), 1188-1194.

Fearon, I.M., Faux, S.P., 2009. Oxidative stress and cardiovascular disease: novel tools give (free) radical insight. Journal of Molecular and Cellular Cardiology 47 372-381.

Gharsallaoui, A., Roudaut, G., Chambin, O., Voilley, A., Saurel, R., 2007. Applications of spray-drying in microencapsulation of food ingredients: an overview. Food Research International 40 (9), 1107-1121.

Gibson, G.R., Kolida, S., 2007. Prebiotic capacity of inulin-type fructans. The Journal of Nutrition 137, 2503S-2506S

Gouin, S., 2004. Microencapsulation: industrial appraisal of existing technologies and trends. Trends in Food Science \& Technology 15 (7-8), 330-347.

Goulas, A.E., Kontominas, M.G., 2007. Combined effect of light salting, modified atmosphere packaging and oregano essential oil on the shelf-life of sea bream (Sparus aurata): biochemical and sensory attributes. Food Chemistry 100 (1), 287-296.

Gourdel, Y., Tronel, J., 2001. Flavoured Gelatin Capsules Capable of Containing Various Substances Used in the Food, Animal Feed, Dietetic and Pharmaceutical Sectors. French Patent Application FR 2807677 A1, Gourdel Tronel Man, France.

Govaris, A., Solomakos, N., Pexara, A., Chatzopoulou, P.S., 2009. The antimicrobial effect of oregano essential oil, nisin and their combination against Salmonella Enteritidis in minced sheep meat during refrigerated storage. International Journal of Food Microbiology 137 (2-3), 175-180.

Gutierrez, J., Barry-Ryan, C., Bourke, P., 2008. The antimicrobial efficacy of plant essential oil combinations and interactions with food ingredients. International Journal of Food Microbiology 124, 91-97.

Jacquot, M., Pernetti, M., 2003. Spray coating and drying processes. In: Nedovic, U., Willaert, R. (Eds.), Cell Immobilization Biotechnology, Series: Focus on Biotechnology. Kluwer Academic Publishers, Dordrecht, pp. 343-356.

Kanakdande, D., Bhosale, R., Singhal, R.S., 2007. Stability of cumin oleoresin microencapsulated in different combination of gum Arabic, maltodextrin and modified starch. Carbohydrate Polymers 67, 536-541.

Kaur, C., Kapoor, H.C., 2001. Antioxidants in fruits and vegetables: the millennium's health. International Journal of Food Science and Technology 703, 725.

Kaushik, V., Roos, Y.H., 2007. Limonene encapsulation in freeze-drying of gum arabic-sucrose-gelatin systems. Lebensmittel-Wissenschaft Und-TechnologieFood Science and Technology 40, 1381-1391.

Kulisic, T., Radonic, A., Katalinic, V., Milos, M., 2004. Use of different methods for testing antioxidative activity of oregano essential oil. Food Chemistry 85 (4) 633-640.

Lambert, R.J.W., Skandamis, P.N., Coote, P.J., Nychas, G.-J.E., 2001. A study of the minimum inhibitory concentration and mode of action of oregano essential oil thymol and cravacrol. Journal of Applied Microbiology 91, 453-462.

Madene, A., Jacquot, M., Scher, J., Desobry, S., 2006. Flavour encapsulation and controlled release - a review. International Journal of Food Science and Technology 41, 1-21.

Minemoto, Y., Adachi, S., Matsuno, R., 1997. Comparison of oxidation of menthyl linoleate encapsulated with gum arabic by hot-air-drying and freeze-drying. Journal of Agricultural and Food Chemistry 45, 4530-4534.

Nastruzzi, C., 1999. Dextran cross-linked gelatin microspheres as a drug delivery system. European Journal of Pharmaceutics and Biopharmaceutics 47 (2), 153160.

Ou, B., Hampsch-Woodill, M., Prior, R.L., 2001. Development and validation of an improved oxygen radical absorbance capacity assay using fluorescein as the fluorescent probe. Journal of Agricultural and Food Chemistry 49, 46194626.

Ou, B., Hampsch-Woodill, M., Flanagam, J., Deemer, E.K., Prior, R.L., Huang, D., 2002. Novel fluorometric assay for hydroxyl radical prevention capacity using fluorescein as the probe. Journal of Agricultural and Food Chemistry 50 2772-2777.

Oussalah, M., Caillet, S., Saucier, I., Lacroix, M., 2007. Inhibitory effects of selected plant essential oils on the growth of four pathogenic bacteria: E. coli 0157:H7 Salmonella Typhimurium, Staphylococcus aureus and Listeria monocytogenes. Food Control 18 (5), 414-420.

Paster, N., Juven, B.J., Shaays, E., Menasherov, M., Nitzan, R., Weisslowicz, H., Ravid U., 1990. Inhibitory effect of oregano and thyme essential oils on molds and foodborne bacteria. Letters in Applied Microbiology 11 (1), 33-37.

Pérez-Alonso, C., Baez-Gonzalez, J.G., Beristain, C.I., Vernon-Carter, E.J., VizcarraMendoza, M.G., 2003. Estimation of the activation energy of carbohydrate polymers blends as selection criteria for their use as wall material for spraydried microcapsules. Carbohydrate Polymers 53, 197-203.

Ponce, A.G., Fritz, R., del Valle, C., Roura, S.I., 2003. Antimicrobial activity of essential oils on the native microflora of organic Swiss chard. Lebensmittel-Wissenschaft Und-Technologie-Food Science and Technology 36, 679-684. 
Pothakamury, U.R., Barbosa-Cánovas, G.V., 1995. Fundamental aspects of controlled release in foods. Trends in Food Science and Technology 6, 397-406.

Prata, A.S., Zanin, M.H.A., Ré, M.I., Grosso, C.R.F., 2008. Release properties of chemical and enzymatic crosslinked gelatin-gum Arabic microparticles containing a fluorescent probe plus vetiver essential oil. Colloids and Surfaces B: Biointerfaces 67 (2), 171-178.

Reuter, S., Gupta, S.C., Chaturvedi, M.M., Aggarwal, B.B., 2010. Oxidative stress inflammation and cancer: how are they linked? Free Radical Biology and Medicine 49, 1603-1616.

Roberfroid, M.B., 2007. Inulin-type fructans: functional food ingredients. The Journal of Nutrition 137, 2493-2502.

Romero-Cano, M.S., Vincent, B., 2002. Controlled release of 4-nitroanisole from poly(lactic acid) nanoparticles. Journal of Controlled Release 82 (1), 127-135.

Saénz, C., Tapia, S., Chávez, J., Robert, P., 2009. Microencapsulation by spray drying of bioactive compounds from cactus pear (Opuntia ficus-indica). Food Chemistry $114,616-622$

Serra, A.T., Duarte, R.O., Bronze, M.R., Duarte, C.M.M., 2011. Identification of bioactive response in traditional cherries from Portugal. Food Chemistry 125, 318-325.

Seydim, A.C., Sarikus, G., 2006. Antimicrobial activity of whey protein based edible films incorporated with oregano, rosemary and garlic essential oils. Food Research International 39 (5), 639-644.

Shu, B., Yu, W., Zhao, Y., Liu, X., 2006. Study on microencapsulation of lycopene by spray-drying. Journal of Food Engineering 76, 664-669.

Skandamis, P., Tassou, C.C., Nychas, G.J.E., 1999. Potential use of essential oils as food preservatives. In: 17th International Symposium of the International
Committee on Food Microbiology and Hygiene (ICFMH), Tuijterlaars, A.C.J., Samson, R.A., Roumbuts, F.M., Notermans, S., (Eds.), Veldhoven, The Netherlands, 13-17 September 1999, pp. 300-303.

Stevenson, D.E., Hurst, R.D., 2007. Polyphenolic phytochemicals - just antioxidants or much more? Cellular and Molecular Life Sciences 64, 2900-2916.

Tari, T.A., Annapure, U.S., Singhal, R.S., Kulkarni, P.R., 2003. Starch-based spherical aggregates: screening of small granule sized starches for entrapment of a model flavouring compound, vanillin. Carbohydrate Polymers 53, 45-51.

Trindade, M.A., Grosso, C.R.F., 2000. The stability of ascorbic acid microencapsulated in granules of rice starch and in gum Arabic. Journal of Microencapsulation 17, $169-176$.

Yoshii, H., Soottitantawat, A., Liu, X.D., Atarashi, T., Furuta, T., Aishima, S., et al. 2001. Flavor release from spray-dried maltodextrin/gum Arabic or soy matrices as a function of storage relative humidity. Innovative Food Science and Engineering Technologies 2, 55-61.

Zhang, Z., Grijpma, D.W., Feijen, J., 2006. Poly(trimethylene carbonate) and monomethoxy poly(ethylene glycol)-block-poly(trimethylene carbonate) nanoparticles for the controlled release of dexamethasone. Journal of Controlled Release 111 (3), 263-270.

Zhao, J., Whistler, R.L., 1994. Spherical aggregates of starch granules as flavor carriers. Food Technology 48, 104-105.

Zhu, X., Xu, S.Y., Wang, Z., 1998. Gelatine as microencapsulating agents for Bcarotene. Food and Fermentation Industries 24, 11-15. 